\title{
Neural Correlates of Craving in Methamphetamine Abuse
}

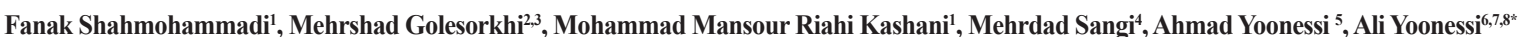

1. Department of Computer Engineering, Islamic Azad University, Tehran North Branch, Tehran, Iran.

2. Translational Neuroscience Program, Institute for Cognitive Science Studies, Tehran, Iran.

3. Neuroimaging and Analysis Group, Research Center for Molecular and Cellular Imaging, Tehran University of Medical Sciences, Tehran, Iran.

4. Auckland Bioengineering Institute, University of Auckland, Auckland, New Zealand.

5. McGill Vision Research, McGill University, Montreal, QC, Canada.

6. Iranian National Center for Addiction Studies, Tehran University of Medical Sciences, Tehran, Iran

7. Brain Mapping Lab, Institute for Cognitive Science Studies, Tehran, Iran.

8. Department of Neuroscience, School of Advanced Technologies in Medicine, Tehran University of Medical Sciences, Tehran, Iran.

Citation: Shahmohammadi, F., Golesorkhi, M., Riahi Kashani, M. M., Sangi, M., Yoonessi, A., \& Yoonessi, A. (2016). Neural correlates of craving in methamphetamine abuse. Basic and Clinical Neuroscience, 7(3), 221-230. http://dx.doi.org/10.15412/J.BCN.03070307

http://dx.doi.org/10.15412/J.BCN.03070307

Article info:

Received: 26 June 2015

First Revision: 09 July 2015

Accepted: 13 January 2016
Key Words:

Event-related potential, Brain, Methamphetamine, Craving

\begin{abstract}
A B S T RA C T
Introduction: Methamphetamine is a powerful psychostimulant that causes significant neurological impairments with long-lasting effects and has provoked serious international concerns about public health. Denial of drug abuse and drug craving are two important factors that make the diagnosis and treatment extremely challenging. Here, we present a novel and rapid noninvasive method with potential application for differentiation and monitoring methamphetamine abuse.

Methods: Visual stimuli comprised a series of images with neutral and methamphetamine-related content. A total of 10 methamphetamine abusers and 10 age-gender matched controls participated in the experiments. Event-related potentials (ERPs) were recorded and compared using a time window analysis method. The ERPs were divided into 19 time windows of $100 \mathrm{~ms}$ with $50 \mathrm{~ms}$ overlaps. The area of positive sections below each window was calculated to measure the differences between the two groups.

Results: Significant differences between two groups were observed from 250 to $500 \mathrm{~ms}$ (P300) in response to methamphetamine-related visual stimuli and 600 to $800 \mathrm{~ms}$ in response to neutral stimuli.

Conclusion: This study presented a novel and noninvasive method based on neural correlates to discriminate healthy individuals from methamphetamine drug abusers. This method can be employed in treatment and monitoring of the methamphetamine abuse.
\end{abstract}

\section{Introduction}

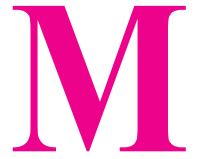

ethamphetamine is an extremely addictive psychostimulant drug that has a direct effect on the monoamine neurotransmitter system of the brain. Its consumption results in the feeling of awareness, high energy, and exhilaration. This substance is mostly popular among young adults, due to its availability, long duration of psychoactive effects, and comparatively low costs (Panenka et al., 2013). Methamphetamine acts

* Corresponding Author:

Ali Yoonessi, $M D, P h D$

Address: Department of Neuroscience, School of Advanced Technologies in Medicine, Tehran University of Medical Sciences, Italy Ave., Tehran, Iran. Tel: +98 (21) 88991118

E-mail:a-yoonessi@tums.ac.ir 
on the dopamine reuptake receptors, and undermines nerves endings by increasing the concentration of dopamine (AresSantos, Granado, \& Moratalla, 2013). Methamphetamine consumption is associated with a considerable neurotoxicity, cognitive disorders, auditory or visual hallucinations, bizarre beliefs, risky behaviors, and psychological problems. These effects can lead to significant social problems which in turn imperil the life of abusers and those around them (McIver et al., 2006; Panenka et al., 2013).

Three basic attributes of methamphetamine abuse revealed by clinical examinations are as follows: 1) Progressive qualitative change in mental symptoms in a sequence of nonpsychotic to a prepsychotic and subsequently a severe psychotic condition; 2) Psychosis due to increased vulnerability; and 3) Chronic vulnerability to relapse (Ujike \& Sato, 2004). In addition, methamphetamine abuse is also a major cause of outbreak in memory disorders (Wyk \& Stuart, 2012) and reduces the accuracy on working memory (Newton et al., 2004).

Crystallized methamphetamine, known in Iran as "Shisheh", is a popular stimulant (Ekhtiari et al., 2010a). An epidemic of methamphetamine consumption has alarmingly increased in Iran and many other parts of the world in recent years (Shariatirad, Maarefvand, \& Ekhtiari, 2013) and many psychiatric emergency services are overwhelmed with patients suffering from methamphetamine-induced psychosis (Fasihpour, Molavi, \& Shariat, 2013; Shariat \& Elahi, 2010). A recent study surveying national drug usage statistics using text messages demonstrated that methamphetamine was the most repeatedly cited drug of choice (Yoonessi \& Ekhtiari, 2013). These facts demonstrate the need for a reliable detection method of methamphetamine abuse.

ERPs or event-related potentials can directly measure neural activity in response to a visual stimulus. These signals are extremely important in measuring the attentional and emotional effects of drug-related stimuli in addiction (Dunning et al., 2011). ERP-based studies on opiate (e.g. heroin) (Franken, Stam, Hendriks, \& Van den Brink, 2003), depressant (e.g. alcohol) (Namkoong, Lee, Lee, Lee, \& An, 2004), and stimulant substances (e.g. cocaine) (Dunning et al., 2011; Van de Laar, Licht, Franken, \& Hendriks, 2004) were effective in differentiating drug abusers and control groups. Comparing ERPs for drug-related visual stimuli with neutral stimuli often results in increased physiological reactions and drug craving in substance abusers.

However, even though global methamphetamine consumption has significantly increased in the past decade, the effects of methamphetamine-related visual stimuli on methamphetamine abusers have not been investigated to our knowledge.
Two well-known studies based on auditory stimuli (Iwanami, Suga, Kato, Nakatani, \& Kaneko, 1993; Iwanami, Suga, Kaneko, Sugiyama, \& Nakatani, 1994) investigated the ERP changes among methamphetamine drug abusers and will be discussed in detail in section 4.1. In addition, few EEG-based studies have shown different quantitative EEG (QEEG) responses between methamphetamine-dependent subjects and controls (Newton et al., 2003; Newton et al., 2004) and between ecstasy-dependent subjects and controls (Dafters, Duffy, O’Donnell, \& Bouquet, 1999). Methamphetamine users often exhibit higher slow wave brain activity than controls, and major differences were observed in delta and theta waves. A detailed review of these studies can be found in Ceballos and colleagues (Ceballos, Bauer, \& Houston, 2009).

Drug craving is one of the most important signatures of methamphetamine abuse. This craving can be defined as an intensive and irresistible desire for drug use, that if it is not complied, redounds to a mental and physical suffering such as asthenia, anorexia, anxiety, insomnia, aggression, and depression (Mokri, Ekhtiari, Edalati, \& Ganjgahi, 2008). Since this intense craving is likely to be correlated with neural activity, it might be detected by neural recording methods such as ERP. Therefore we aimed to utilize ERPs to detect this craving in our proposed method.

Diagnosis of Methamphetamine abuse is much more challenging compared to other substances such as heroin or alcohol. Complications arise from the fact that the current biochemical tests often fail to diagnose the methamphetamine abuse after a short break in drug consumption (the half-life of methamphetamine varies between 10 and 30 hours); the detection time limitation of methamphetamine abuse has been elaborately discussed by Verstraete (2004). Hence, a noninvasive test that can reliably discriminate between methamphetamine abusers and controls can be extremely useful. A reliable method also needs to be subconscious in order to avoid conscious denial attempts by the drug user. We believe that ERPs are suited the best for this purpose.

Here we provide a method to discriminate between methamphetamine abusers and normal healthy subjects. Our method can effectively differentiate between abusers and controls with a simple fast noninvasive procedure. Furthermore, the effect can be potentially robust to denial attempts by the user

\section{Methods}

\subsection{Visual stimuli}

Methamphetamine-related and neutral images selected from freely available online sources (Ekhtiari, Alam-Meh- 
rjerdi, Nouri, George, \& Mokri, 2010b) were employed as stimuli. All images were color photographs with a resolution of $1754 \times 1240$ pixels depicted on a black background. These images contained 7 categories, and were divided into 3 major types: 1) Drug-associated cues; 2) Drugs (including 3 categories: 2-1-drug; 2-2-drug with face; 2-3- drug with hand); 3) Neutrals (including 3 categories: 3-1- neutral; 3-2- neutral with face; 3-3- neutral with hand). Sample images for each of these categories are represented in Figure 1. Every category included 13 images, and each image was shown only once in a fixed pre-randomized order. The stimuli were shown on a 17-inch screen of a LCD monitor (Samsung, South Korea, SyncMaster SN1943, 1360×768 pixels, $60 \mathrm{HZ}$ ) which was gamma calibrated (mean luminance of $56.2 \mathrm{~cd} / \mathrm{m}^{2}$ ). Each image was presented for a period of $500 \mathrm{~ms}$ followed by an inter-trial interval of $1000 \mathrm{~ms}$, during which the screen was black. In total, each subject viewed 91 images, and the total experiment time was about 3 minutes.

\subsection{Subjects}

Ten methamphetamine drug abusers and 10 age-gender matched controls participated voluntarily in this study. All participants were male with the age range of 18 to 40 years (mean age: $28.71 \pm 7.11$ ) without history of comorbidity, head injury, or trauma. The selection criteria for abuser group were being pure methamphetamine abusers, not consuming any other substances except cigarettes, and using methamphetamine continuously for at least 6 months. The results of their urine test strips (ACON Labs, CA, USA; ABON Biopharm, Hangzhou, China) were positive, and they were abstinent from any drugs for minimum of 7 days prior to the experiment.

Table 1 summarizes the drug history of all participants in this study. Prior to the recording session, the aims and procedures of the experiment were briefly explained. All subjects gave prior consent to participation in the experiments, and the subjects were aware of the purpose of the experiment. Personal information and risky behavior backgrounds were assessed by filling a questionnaire. All subjects had normal or corrected to normal vision. All experimental procedures followed university's ethical guidelines and adhered to the Declaration of Helsinki.

\subsection{Experimental procedures}

The visual stimulus was presented on the monitor screen viewed from a distance of $50 \mathrm{~cm}$. Head positions were fixed using a chin rest. Subjects were instructed to maintain fixation while viewing the stimulus passively in a dark room.

An electroencephalograph (EEG) cap with 32 channels was attached to the subject's scalp, and the conductive gel was injected. The subjects were instructed to avoid body or jaw movements, maintain normal breathing and heart rhythms, and to limit blinking during the test to the extent possible.

Each subject was tested in 2 separate blocks with a short break between the first and the second block. The second recording was performed to improve recording quality in case the signal-to-noise in the first recording was not sufficient. From two recordings the one with the highest signal-to-noise ratio was used for subsequent analysis.

ERP recordings were collected using ANT EEG/ERP recording system (A.N.T. Enschede, Netherlands) with 32 channels ANT cap (Wave Guard cap system, Netherlands), which was positioned according to the 10-20 system. Electrode impedances were at or below $1 \mathrm{k} \Omega$ for all electrodes. The experimental task was created using the "eevoke" software (A.N.T. Enschede, Netherlands). The raw data was subsequently transferred to MATLAB (Mathworks, USA) using the ASA signal processing software (A.N.T. Enschede, Netherlands). Sampling rate was set at $512 \mathrm{~Hz}$, and mastoid electrodes were chosen as the reference electrodes. The designated ground electrode in the front of the cap was employed as electrical ground.

Software digital filtering was performed in MATLAB using EEGLAB toolbox (Delorme \& Makeig, 2004) functions. A $50 \mathrm{~Hz}$ notch filtering scheme was employed to remove the $\mathrm{AC}$-induced noise followed by a band-pass filtering (Using the pop_eegfilt function in EEGLAB toolbox) between 0.5 and $45 \mathrm{~Hz}$ to remove the probable low and high frequency artifacts in the EEG signals. The baseline noise was removed (using first $100 \mathrm{~ms}$ as basis) on the continuous EEG. The extracted epochs were $1200 \mathrm{~ms}$ long (200 ms before stimulus onset and $1000 \mathrm{~ms}$ after onset) and the average removal was performed on each epoch. The acceptable amplitude threshold was limited to $\pm 70 \mu \mathrm{V}$ in order to remove EOG artifacts. In each epoch, if the number of amplitudes over the threshold exceeded more than 50, the whole epoch was discarded and excluded from further analysis.

\subsection{Analysis}

All signal processing procedures were performed using MATLAB and EEGLAB toolbox (Delorme \& Makeig, 2004). ERPs for each subject were constructed by averaging epochs based on all 7 categories and 3 types of images, and subsequently the grand averages were calculated across all subjects in each subject group.

The difference of grand averaged ERPs based on 7 categories of images was calculated in 32 channels (drug users minus controls) in order to find the best channels. Then, the 
area below difference curves (absolute values of amplitudes in each curve were considered for area calculation) was calculated. Previous studies on craving for cocaine, opiate, and alcohol suggest that dorsolateral prefrontal cortex is significantly activated during craving episodes (Garavan et al., 2000; Brody, Mandelkern, London, Childress, Lee, \& Bota, et al., 2002). Thus, it is likely that in our study dorsolateral prefrontal electrodes show significant activation in response to induced craving from visual stimulus. Therefore, the area values of 7 categories in each channel were averaged and the channels FPz, FP2, F7, T8, P8, and Oz with the highest signal-to-noise ratio were selected as representatives of brain activity. These channels demonstrated significant differences between drug users and controls. Among the 6 selected channels, the recorded reactions of FPz were the most significant and thus FPz channel was selected as the major channel of interest.

Analysis was divided into two steps. In the first step, we compared grand averaged ERPs of 10 drug users and 10 controls in response to 7 categories of images. Among these categories, drug-related images (drug, drug with face, and drug with hand) showed significant differences (Figure 2); additionally we compared scalp topographies of 10 drug users and 10 controls in response to 3 types of images (Figure 3 ).

In the second step, we extracted differentiating features between drug users and controls in response to 3 types of images. Each group (drug users and controls) was divided into two subgroups. The first subgroup of drug users contained the ERPs of first 5, and the second contained the ERPs of second 5 subjects (similarly for controls). Features were constructed using the first subgroups for both drug users and controls and was subsequently validated on the second subgroups. Several standard methods are employed for ERP quantification (Hoormann, Falkenstein, Schwarzenau, \& Hohnsbein, 1998; Luck, 2012). Here, we employed window analysis method to find significant differentiating features between drug users and controls. This quantification procedure was performed in 3 stages:

First stage: The grand averaged ERPs based on 3 types of images (with $1000 \mathrm{~ms}$ time range after stimulus onset) were divided into 19 windows of $100 \mathrm{~ms}$ with $50 \mathrm{~ms}$ overlaps; Thus, each window started after the half window time of the former window elapsed.

Second stage: The area of positive sections in each window was calculated and plotted in channel FPz, represented in Figure 4.

Third stage: The results for drug users and controls were compared among 3 types of images to find the window num- bers with significant difference. Subsequently, statistical significance of windows between drug user and control groups was assessed by ANOVA2 ( $\mathrm{P}<0.01$ as significant) for each type of image.

\section{Results}

\subsection{Identifying appropriate leads}

Figure 2 shows the grand averaged ERPs for 10 drug users in comparison with 10 controls in response to 3 categories of images. The results demonstrate significant positive reactions in response to drug-related stimuli (Categories: drug, drug with face, and drug with hand), which are most pronounced as P300 peaks in time range from about $300 \mathrm{~ms}$ to $600 \mathrm{~ms}$. Furthermore, the scalp topographies of drug users and control group were plotted based on 3 types of images (drug-associated cues, drugs, and neutrals) in 3 time ranges of $300 \mathrm{~ms}$, indicating significant activities of the drug users in response to "drugs" type, which were maximal in FP1, FPz, FP2, and F8 in time range from 300 to $600 \mathrm{~ms}$ (Figure 3).

\subsection{Differentiating features}

Features extracted from 3 types of images were as follows:

\subsubsection{Drug-associated cues}

Figure 4a shows the windowing results for "drug-associated cues" type in which the numbers $8,9,10,11$, and 12 indicate significant $(\mathrm{P}<0.001)$ differences between drug users and controls. The time of these windows ranges from 350 to $650 \mathrm{~ms}$. Thresholds were specified in each type of images which used in proposed algorithm as condition. The minimum threshold of this type was set at $55 \mu \mathrm{V}$.

\subsubsection{Drugs}

Figure $4 \mathrm{~b}$ shows the windowing results for "drugs" type in which the numbers $6,7,8$, and 9 indicate significant differences $(\mathrm{P}<0.001)$ between drug users and controls. The time of these windows ranges from 250 to $500 \mathrm{~ms}$, and minimum threshold was set at $60 \mu \mathrm{V}$.

\subsubsection{Neutrals}

Figure $4 \mathrm{c}$ shows the windowing results for "neutrals" type in which the numbers 13, 14, and 15 indicate significant $(\mathrm{P}<0.001)$ differences between drug users and controls. The time range of these windows is from 600 to $800 \mathrm{~ms}$, and minimum threshold was set at $70 \mu \mathrm{V}$.

Following extraction of differentiating features and specifying the minimum value of threshold, values of threshold were 


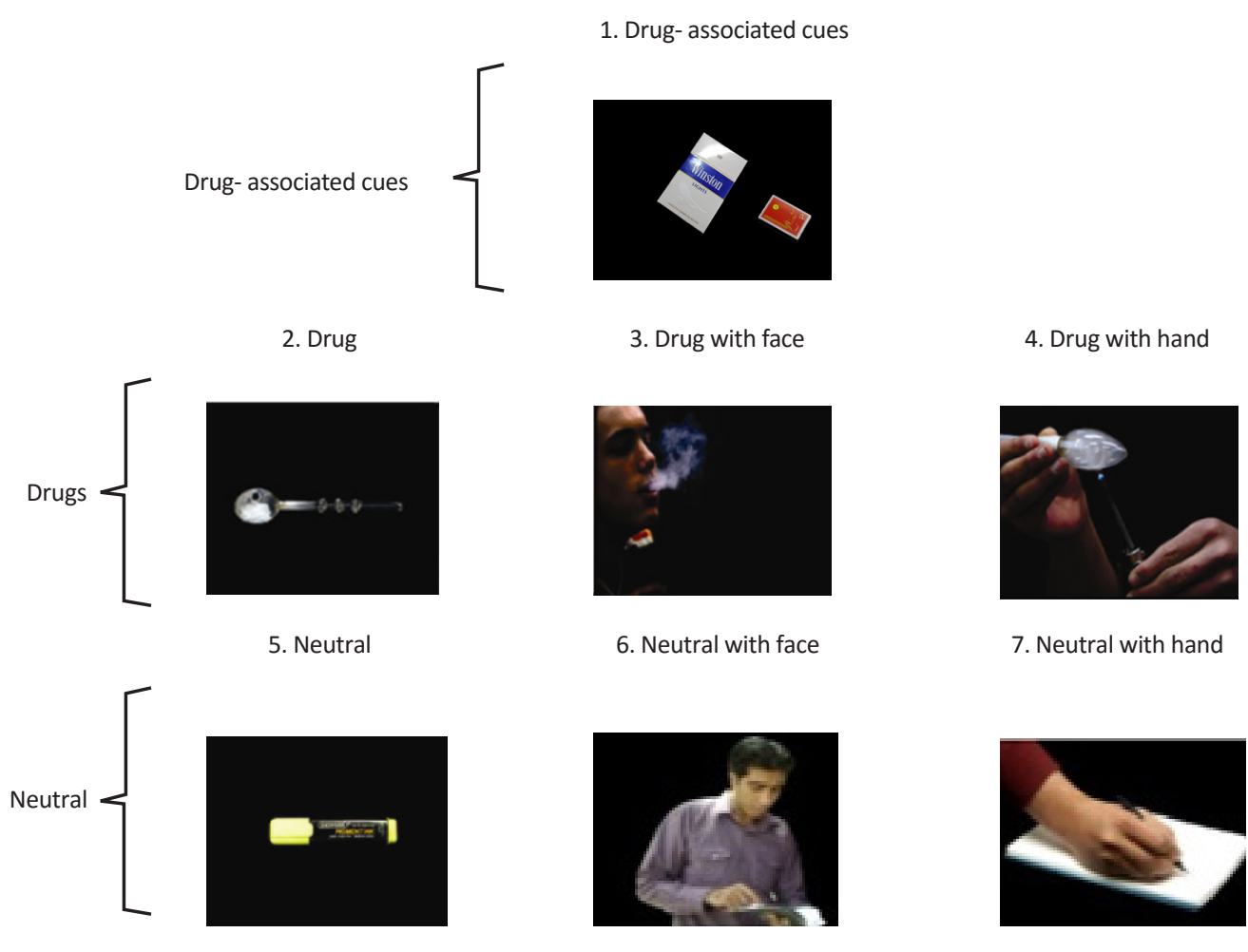

NEUR:SCIENCE

Figure 1. Examples of visual stimuli for each 7 categories (1. drug-associated cues, 2. drug, 3. drug with face, 4. drug with hand, 5. neutral, 6. neutral with face, 7. neutral with hand), which were divided into 3 major types (1. drug-associated cues (top row), 2. drugs (middle row), and 3. neutrals (bottom row).

adjusted in order to reach to the highest diagnostic accuracy (see the pseudocode of the algorithm in the appendix).

The extracted algorithm was subsequently tested on second subgroups. The results of test were accurate for 4 out of 5 drug users (corresponds to $80 \%$ accuracy) and for 4 out of 5 controls (corresponds to $80 \%$ accuracy).

\section{Discussion}

In this study, we presented a novel noninvasive method based on ERPs analysis to differentiate the healthy individuals from methamphetamine drug abusers. This method can be employed in monitoring of methamphetamine abuse treatment.

Our results indicated that the drug users showed higher brain activities in response to methamphetamine-related stimuli in time range from about $300 \mathrm{~ms}$ to $600 \mathrm{~ms}$ (as P300 peaks), which were maximal in channels FP1, FPz, FP2, and F8. Furthermore, the window analysis showed the significant differences between drug users and control group in time ranges from 350 to $650 \mathrm{~ms}$ (in response to drug-associated stimuli), 250 to $500 \mathrm{~ms}$ (in response to drugs stimuli), and 600 to $800 \mathrm{~ms}$ (in response to neutrals stimuli), which were employed as differentiating features.

\subsection{Comparing with previous ERP based methods}

ERPs-based studies are useful in studying perception and attention due to the extreme time resolution and comparatively low cost. Also, they are useful in diagnosis of mental disorders such as depression (Kalatzis et al., 2003), multiple sclerosis (Slater, Wu, Honig, Ramsay, \& Morgan, 1994), and schizophrenia (Vasios, Papageorgiou, Matsopoulos, Nikita, \& Uzunoglu, 2002). ERP-based studies of other substances such as alcohol (Namkoong et al., 2004), heroin

Table 1. Summary of drug use history for the methamphetamine users and controls.

\begin{tabular}{|c|c|c|}
\hline & Methamphetamine abusers $(\mathbf{n}=10)$ & Controls $(\mathbf{n}=10)$ \\
\hline History of using other substances & - & - \\
\hline History of cigarette smoking (current/never or tried) & $10 / 0$ & $0 / 10$ \\
\hline
\end{tabular}




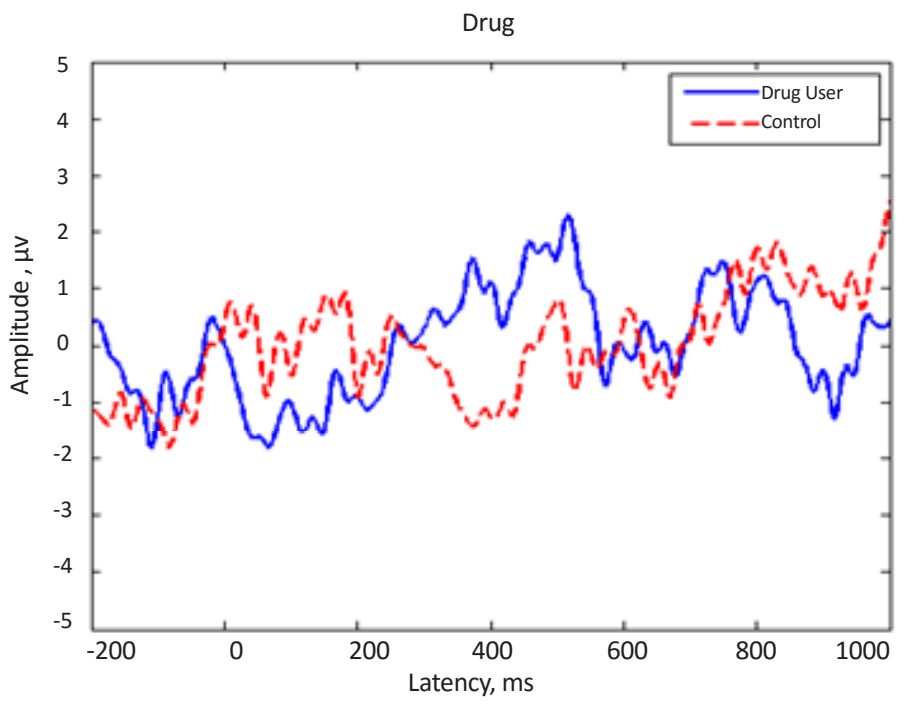

a)

Drug with face

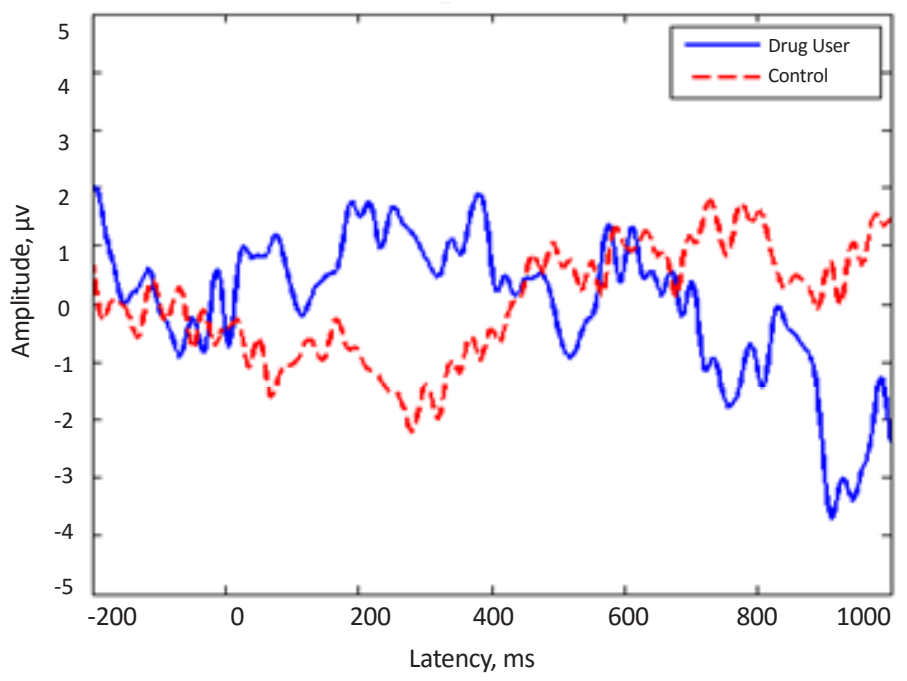

Drug with hand

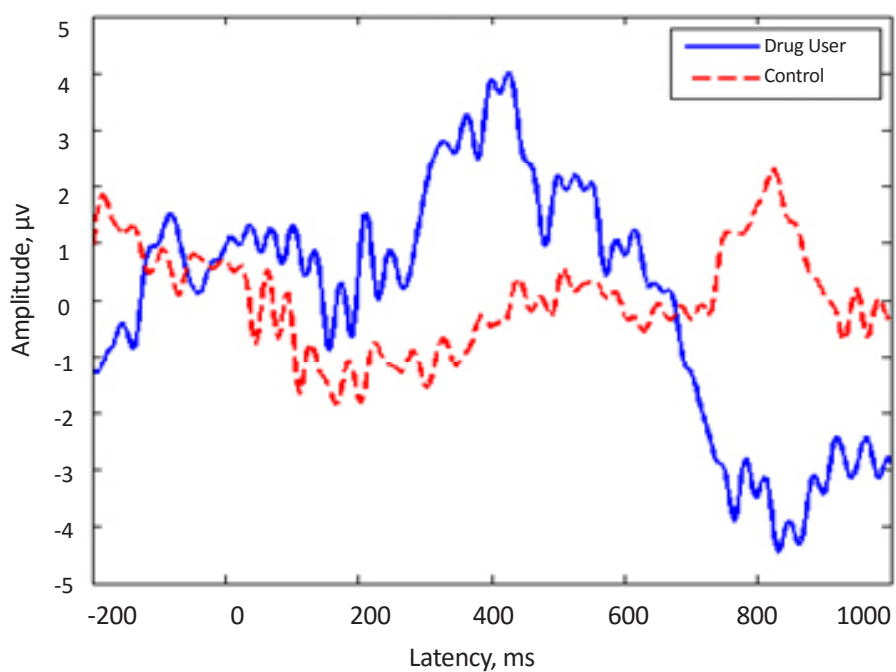

NEUR:SCIENCE

Figure 2. Comparison of grand averaged ERPs for 10 drug users and 10 controls in response to 3 categories of images: a) Drug, b) Drug with face, and c) Drug with hand, channel: FPz. Stimulus onset occurred at $0 \mathrm{~ms}$. The biggest difference between drug users and controls are observed in drug with hand category. 
(Franken et al., 2003), and cocaine (Dunning et al., 2011; Van de Laar et al., 2004; Franken, Hulstijn, Stam, Hendriks, \& Van den Brink, 2004) revealed significant differences between drug abusers and controls based on the effect of visual stimuli. In particular, Van de Laar et al. (2004) found differential ERP responses to drug-related visual stimuli between cocaine users and controls, where drug users exhibited larger N300 than normal. Franken et al. (2004) found larger positive slow wave ERP responses in drug users with high cravings following cocaine-related visual stimuli.

However, even though ERPs have been employed in studies of many other substances, employment of ERP in methamphetamine abuse has been extremely limited thus far. Furthermore, to our knowledge, no previous study investigated the effects of methamphetamine-related visual stimuli on methamphetamine-dependents. However, a few studies investigated ERP changes related to auditory stimuli in methamphetamine users. Iwanami et al. (1993) examined methamphetamine psychosis using an auditory discrimination task and found that negative components area became smaller, and P300 components were delayed. However their study, unlike ours, did not find significant differences in P300 amplitudes between drug users and controls. A follow up study by the same group (Iwanami et al., 1994) found significant differences in P300 amplitudes between methamphetamine psychotics and controls in the $\mathrm{Fz}$ and $\mathrm{Cz}$ electrodes, similar to our results where we found maximum P300 amplitude differences in FPz electrode. In addition, brain imaging studies of methamphetamine abusers have found decreased cerebral blood flow in putamen cortices and right lateral parietal cortex, but increased blood flow in the left temporo-parietal white matter, left occipital regions, and right posterior parietal regions (Chang et al., 2002). These changes in cerebral blood flow might be correlated with our findings of changes in P300 amplitudes in methamphetamine users.

Many methamphetamine users often employ methods to negate the result of biochemical tests and deny their addiction. Therefore, using biochemical tests without additional measures might be unreliable in these cases. Since our algorithm is based on subconscious craving, it is potentially robust to denial attempts and can be employed as a complementary method along with standard biochemical tests. This method can also be used to monitor effectiveness of therapy and the possible relapses. Also, the subsequent changes in the ERPs can be an indicator of treatment success. Furthermore, due to the ongoing reduction in cost of EEG systems and the availability of commercial systems aimed for human computer interaction, it might be possible to employ this method to monitor drug users at home in the future.

\subsection{Possible limitations}

Since the consumption methods of other substances such as ecstasy, heroin, and cocaine are very similar to methamphetamine (e.g. smoking, snorting, and injecting), and the content of the drug-related category of visual images were not methamphetamine specific in all images, it might be possible that images related to other drugs result in the same ERP patterns in methamphetamine users. However, since the specific changes in the brain as a result of abuse of other substanc-

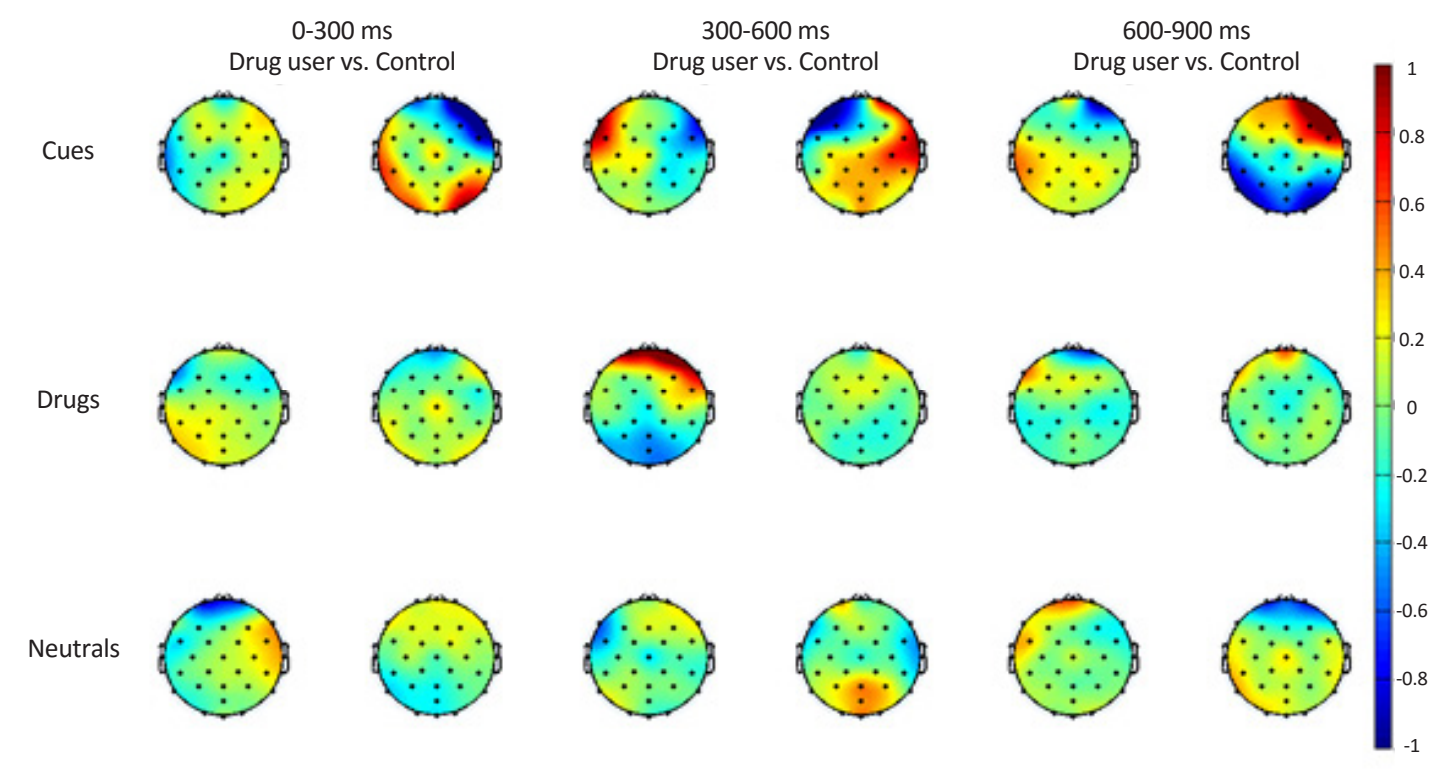

NEUR:SCIENCE

Figure 3. Scalp topographies of drug user versus control group in response to 3 types of images: Drug-associated cues (top row), drugs (middle row), and neutrals (bottom row), in 3 time ranges of $300 \mathrm{~ms}$. 


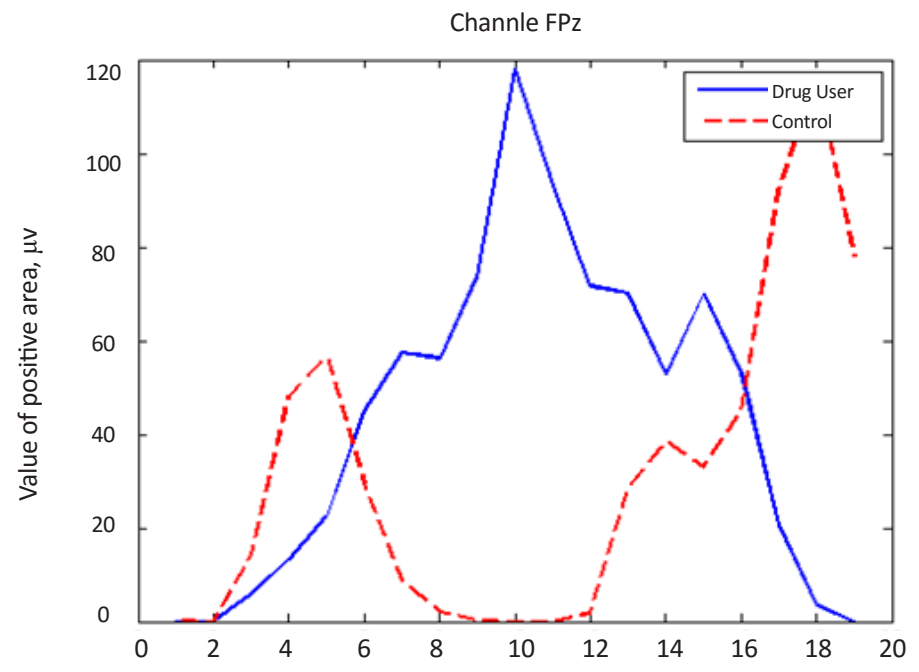

a)

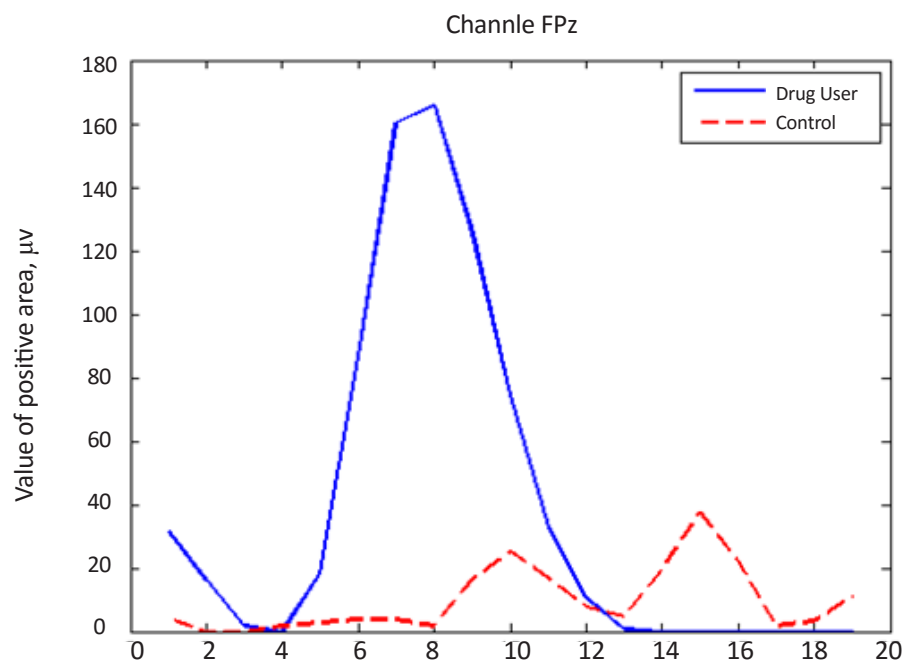

b)

Channle FPz

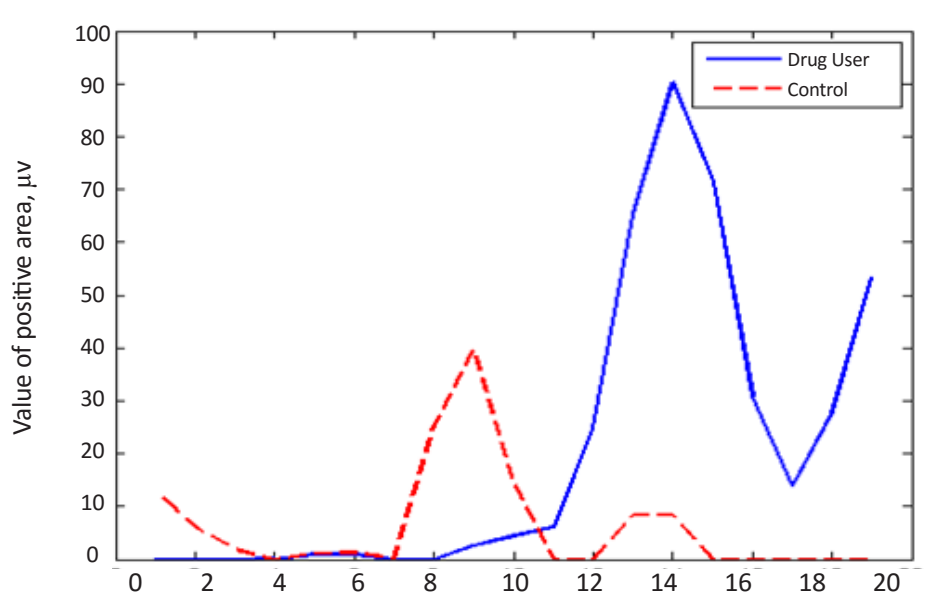

NEUR SCIENCE

Figure 4. Time window analysis for the first subgroups of drug users and controls in response to 3 types of images: a) Drugassociated cues, b) Drugs, and c) Neutrals. 
es such as heroin are different, it is unlikely that the heroin abusers demonstrate identical changes in P300 components. However, a more serious concern is nonspecificity for the target drug. This issue can probably give rise to false positive results for drug users that consume other types of addictive substances particularly those considered as stimulants.

Comparing our results with previous results for other substances such as heroin or cocaine, we found that changes in P300 component are often different for those substances. Nevertheless, our algorithm is intended to be employed complementary to standard biochemical tests, and therefore this issue might be less important if the algorithm is employed in addition to standard tests. Another possible limitation in this study was the small number of methamphetamine drug abusers. The limitation arose from the criteria to recruit the subjects who were only recruited if they were only using methamphetamine (except smoking that was inevitable between them), which reduced the possible available sample pool significantly.

In this paper, we provided a novel and noninvasive method for differentiation of methamphetamine addiction. This method is inherently robust to possible denial attempts and can potentially be more accurate than the common biochemical tests. This method can be employed in discrimination and treatment monitoring of methamphetamine addiction.

\section{Appendix: Pseudocode of the algorithm}

\section{Input: Raw EEG of subject}

Output: Positive or negative results for methamphetamine abuse

\section{Load raw EEG of selected subject.}

- EEG preprocessing includes frequency filtering, baseline noise removal, epochs extracting, average removal for each epoch, and EOG artifact removing.

- ERPs constructing based on 3 types of images (associated cues, drugs, and neutrals).

- Dividing ERPs into 19 time windows of $100 \mathrm{~ms}$ with 50 ms overlaps.

- Calculating the area of positive sections below each window.

- In channel FPZ:

- If (in "drugs" type, the value of positive area of windows with numbers: 6|| 7|| 8|| $9>60$ ) || (in "neutrals" type, the value of positive area of window with number: $14>70$ )
- disp ('The result of test is positive, the subject is a drug user');

- else

- disp ('The result of test is negative, the subject is not a drug user');

- end

\section{Acknowledgements}

This study was supported by Iranian National Center for Addiction Studies, Tehran University of Medical Sciences, and Institute for Cognitive Science Studies.

\section{Conflict of Interest}

This is to certify that authors declared no conflict of interest.

\section{References}

Ares-Santos, S., Granado, N., \& Moratalla, R. (2013). The role of dopamine receptors in the neurotoxicity of methamphetamine. Journal of Internal Medicine, 273(5), 437-453. doi: 10.1111/joim.12049

Brody, A. L., Mandelkern, M. A., London, E., Childress, A. R., Lee, G. S., Bota, R. G., et al. (2002). Brain metabolic changes during cigarette craving. Archives of General Psychiatry, 59(12), 1162-1172. doi: 10.1001/archpsyc.59.12.1162

Ceballos, N. A., Bauer, L. O., \& Houston, R. J. (2009). Recent EEG and ERP Findings in Substance Abusers. Clinical EEG and Neuroscience, $40(2), 122-128$

Chang, L., Ernst, T., Speck, O., Patel, H., DeSilva, M., Leonido-Yee, M., et al. (2002). Perfusion MRI and computerized cognitive test abnormalities in abstinent methamphetamine users. Psychiatry Research: Neuroimaging, 114(2), 65-79.

Dafters, R. I., Duffy, F., O'Donnell, P. J., \& Bouquet, C. (1999). Level of use of 3, 4-methylenedioxymethamphetamine (MDMA or Ecstasy) in humans correlates with EEG power and coherence. Psychopharmacology, 145(1), 82-90.

Delorme, A., \& Makeig, S. (2004). EEGLAB: an open source toolbox for analysis of single-trial EEG dynamics including independent component analysis. Journal of Neuroscience Methods, 134(1), 9-21.

Dunning, J. P., Parvaz, M. A., Hajcak, G., Maloney, T., Alia-Klein, N., Woicik, P. A., et al. (2011). Motivated attention to cocaine and emotional cues in abstinent and current cocaine users: An ERP study. European Journal of Neuroscience, 33(9), 1716-1723.

Ekhtiari, H., Alam-Mehrjerdi, Z., Hassani-Abharian, P., Nouri, M., Farnam, R., \& Mokri, A. (2010a). Examination and evaluation of craving-inductive verbal cues among Persian-speaking methamphetamine abusers. Advances in Cognitive Science, 12(2), 69-82.

Ekhtiari, H., Alam-Mehrjerdi, Z., Nouri, M., George, S., \& Mokri, A. (2010b). Designing and evaluation of reliability and validity of vis- 
ual cue-induced craving assessment task for methamphetamine smokers. Basic and Clinical Neuroscience, 1(4), 33-46.

Fasihpour, B., Molavi, S., \& Shariat, S. V. (2013). Clinical features of inpatients with methamphetamine-induced psychosis. Journal of Mental Health, 22(4), 341-349. doi: 10.3109/0963823.2012.745184

Franken, I. H., Hulstijn, K. P., Stam, C. J., Hendriks, V. M., \& Van den Brink, W. (2004). Two new neurophysiological indices of cocaine craving: Evoked brain potentials and cue modulated startle reflex. Journal of Psychopharmacology, 18(4), 544-552. doi: $10.1177 / 0269881104047282$

Franken, I. H., Stam, C. J., Hendriks, V. M., \& Van den Brink, W (2003). Neurophysiological evidence for abnormal cognitive processing of drug cues in heroin dependence. Psychopharmacology, 170(2), 205-212. doi: 10.1007/s00213-003-1542-7

Garavan, H., Pankiewicz, J., Bloom, A., Cho, J. K., Sperry, L., Ross, T. J., et al. (2000). Cue-induced cocaine craving: Neuroanatomical specificity for drug users and drug stimuli. American Journal of Psychiatry, 157(11), 1789-1798.

Hoormann, J., Falkenstein, M., Schwarzenau, P., \& Hohnsbein, J. (1998). Methods for the quantification and statistical testing of ERP differences across conditions. Behavior Research Methods, Instruments, and Computers, 30(1), 103-109. doi: 10.3758/.BF03209420

Iwanami, A., Suga, I., Kaneko, T., Sugiyama, A., \& Nakatani, Y. (1994). P300 component of event-related potentials in methamphetamine psychosis and schizophernia. Progress in Neuro-Psychopharmacology and Biological Psychiatry, 18(3), 465-475.

Iwanami, A., Suga, I., Kato, N., Nakatani, Y., \& Kaneko, T. (1993). Event-related potentials in methamphetamine psychosis during an auditory discrimination task. European Archives of Psychiatry and Clinical Neuroscience, 242(4), 203-208.

Kalatzis, I., Piliouras, N., Ventouras, E., Papageorgiou, C. C., Rabavilas, A. D., \& Cavouras, D. (2003). Design and implementation of an SVM-based computer classification system for discriminating depressive patients from healthy controls using the P600 component of ERP signals. Computer Methods and Programs in Biomedicine, 75(1), 11-22.

Luck, S. J. (2012). Event-related potentials. In H. Cooper, D. L Long, A. T. Panter, D. Rindskopf \& K. J. Sher (Eds.), APA Handbook of Research Methods in Psychology (Vol. 1, pp. 523-46). Washington, D.C.: American Psychological Association.

McIver, C., McGregor, C., Baigent, M., Spain, D., Newcombe, D., \& Ali, R. (2006). Guidelines for the medical management of patients with methamphetamine-induced psychosis. South Australia: Drug and Alcohol Services.

Mokri, A., Ekhtiari, H., Edalati, H., \& Ganjgahi, H. (2008). Relationship between degree of craving and different dimensions of addiction severity in heroin intravenous users. Iranian Journal of Psychiatry and Clinical Psychology, 14(3), 298-306.

Namkoong, K., Lee, E., Lee, C. H., Lee, B. O., \& An, S. K. (2004) Increased P3 amplitudes induced by alcohol related pictures in patients with alcohol dependence. Alcoholism-Clinical and Experimental Research, 28(9), 1317-1323. doi: 10.1097/01. ALC.0000139828.78099.69

Newton, T. F., Cook, I. A., Kalechstein, A. D., Duran, S., Monroy, F., Ling, W., et al. (2003). Quantitative EEG abnormalities in recently abstinent methamphetamine dependent individuals. Clinical Neurophysiology, 114(3), 410-415.
Newton, T. F., Kalechstein, A. D., Hardy, D. J., Cook, I. A., Nestor, L., \& Leuchter, A. F. (2004). Association between quantitative EEG and neurocognition in methamphetamine-dependent volunteers. Clinical Neurophysiology, 115(1), 194-198.

Panenka, W. J., Procyshyn, R. M., Lecomte, T., Macewan, G. W., Flynn, S. W., Honer, W. G., et al. (2013). Methamphetamine use: A comprehensive review of molecular, preclinical and clinical findings. Drug and Alcohol Dependence, 129(3), 167-179.

Shariat, S. V., \& Elahi, A. (2010). Symptoms and course of psychosis after methamphetamine abuse: One-year follow-up of a case Primary Care Companion to the Journal of Clinical Psychiatry, 12(5), PCC.10|00959. doi: 10.4088/PCC.10100959gry

Shariatirad, S., Maarefvand, M., \& Ekhtiari, H. (2013). Emergence of a methamphetamine crisis in Iran. Drug and Alcohol Review, 32(2), 223-224. doi: 10.1111/dar.12014

Slater, J. D., Wu, F. Y., Honig, L. S., Ramsay, R. E., \& Morgan, R. (1994). Neural network analysis of the P300 event-related potential in multiple sclerosis. Electroencephalography and Clinical Neurophysiology, 90(2), 114-122.

Ujike, H., \& Sato, M. (2004). Clinical features of sensitization to methamphetamine observed in patients with methamphetamine dependence and psychosis. Annals of the New York Academy of Sciences, 1025(1), 279-287.

Van de Laar, M. C., Licht, R., Franken, I. H., \& Hendriks, V. M. (2004). Event-related potentials indicate motivational relevance of cocaine cues in abstinent cocaine addicts. Psychopharmacology, 177(1-2), 121-129.

Vasios, C., Papageorgiou, C., Matsopoulos, G. K., Nikita, K. S., \& Uzunoglu, N. (2002). A decision support system of evoked potentials for the classification of patients with first-episode schizophrenia. German Journal of Psychiatry, 5, 78-84

Verstraete, A. G. (2004). Detection times of drugs of abuse in blood, urine, and oral fluid. Therapeutic Drug Monitoring, 26(2), 200-205.

Wyk, C. V., \& Stuart, A. D. (2012). A comparative study of the effects of methamphetamine on memory in existing and recovering addicts from a South African population: Original research. Journal of Interdisciplinary Health Sciences, 17(1), 9. doi: 10.4102/hsag.v17i1.607

Yoonessi, A., \& Ekhtiari, H. (2013). Text messages as a tool for assessing public concern about drug problems. International Journal on Drug Policy, 24(6), 624-627. doi: 10.1016/j.drugpo.2013.06.002 